\title{
Wavelet multilevel augmentation method for linear boundary value problems
}

\author{
Somlak Utudee ${ }^{1}$ and Montri Maleewong ${ }^{2^{*}}$
}

\author{
*Correspondence: \\ Montri.M@ku.ac.th \\ ${ }^{2}$ Department of Mathematics, \\ Faculty of Science, Kasetsart \\ University, Bangkok, 10900, Thailand \\ Full list of author information is \\ available at the end of the article
}

\begin{abstract}
This work presents a new approach to numerically solve the general linear two-point boundary value problems with Dirichlet boundary conditions. Multilevel bases from the anti-derivatives of the Daubechies wavelets are constructed in conjunction with the augmentation method. The accuracy of numerical solutions can be improved by increasing the number of basis levels, but the computational cost also increases drastically. The multilevel augmentation method can be applied to reduce the computational time by splitting the coefficient matrix into smaller submatrices. Then the unknown coefficients in the higher level can be solved separately. The convergent rate of this method is $2^{s}$, where $1 \leq s \leq p+1$, when the anti-derivatives of the Daubechies wavelets order $p$ are applied. Some numerical examples are also presented to confirm our theoretical results.
\end{abstract}

MSC: $65 J 10 ; 65 \mathrm{~L} 10$

Keywords: wavelets; multilevel augmentation method; boundary value problems; Dirichlet boundary conditions

\section{Introduction}

Boundary value problems can be viewed as mathematical models in science and engineering. For real world applications, exact solutions are not available. Numerical methods are required to solve numerically the models. The efficient methods provide approximate solutions by choosing the appropriate subspaces of solution spaces and their suitable bases. By applying suitable formulations, the linear model equation can be discretized to a linear system. A more accurate approximation can be obtained by increasing the number of basis functions. However, this leads to a larger discretized linear system. To save the computational cost, one can use the multilevel augmentation method. The resulting coefficient matrix corresponding to the finer level of approximate spaces is obtained by augmenting a matrix corresponding to a coarser level. Instead of solving the linear system at the finer level, the coefficient matrix can be separated so that the smaller system at the coarser level can be taken. Thus, the additional computational cost is proportional to the dimension of the different space between the spaces of the finer level and the coarser level, not the dimension of the whole finer level. This method allows us to develop faster and accurate algorithms for solving differential equations (see, e.g., [1-3], and [4]). These previous researches considered the second kind of equations and constructed piecewise polynomials as bases for the subspaces of the Sobolev spaces $H_{0}^{m}(0,1)$ consisting of elements satisfying

(c) 2015 Utudee and Maleewong; licensee Springer. This article is distributed under the terms of the Creative Commons Attribution 4.0 International License (http://creativecommons.org/licenses/by/4.0/), which permits unrestricted use, distribution, and reproduction in any medium, provided you give appropriate credit to the original author(s) and the source, provide a link to the Creative Commons license, and indicate if changes were made. 
the homogeneous boundary conditions

$$
u^{(j)}(0)=u^{(j)}(1)=0, \quad \text { for } j=0,1, \ldots, m-1 .
$$

On the other hand, wavelets can be applied to discretize differential equations (see, e.g., $[5,6])$. Related numerical methods with the applications of Haar and Legendre wavelets for solving boundary value problem are proposed by Siraj-ul-Islam et al. [7, 8]. The advantage of wavelet basis is its capability to approximate solutions of differential equations. The wavelet Galerkin method is one of the most powerful methods that can be used to solve ordinary and partial differential equations (see, e.g., [9-11], and [12]). In addition, the accuracy of the approximate solutions can easily be improved by merely increasing the numbers of wavelet basis functions and the orders of wavelets. However, the wavelet basis is not straightforwardly adjusted to satisfy general boundary conditions. In 1992, Xu and Shann introduced a different approach to handle the boundary conditions by using the anti-derivatives of Daubechies wavelets [13]. These anti-derivatives form bases for the finite-dimensional subspaces of Sobolev space $H^{1}$ and are used to construct an algorithm for approximating solutions.

In this work, we propose the method that combines the main advantages of wavelet bases and multilevel augmentation together. That is, we apply the multilevel augmentation of operators in conjunction with the anti-derivatives of Daubechies wavelets to approximate linear differential equations in the case of Dirichlet boundary conditions. The originality of this work is that we introduce the anti-derivatives of Daubechies wavelets for solving linear boundary value problems (see [13]) and apply this basis type with the augmentation method proposed by Chen (see, e.g., [1-3] and [4]). By this concept, we obtain a new approach to reduce the computational time for solving the linear system resulting from discretizing a linear differential equation.

Given the interval $\Omega:=(0, R)$, we use the notation $L^{2}(\Omega)$ to denote the space of square integrable functions on $\Omega$ with standard inner product (., .) defined by

$$
(u, v)=\int_{0}^{R} u(x) v(x) d x
$$

and the associated norm $\|\cdot\|$.

Let $H^{s}(\Omega)$ denote the standard Sobolev space with the norm $\|\cdot\|_{s}$ given by

$$
\|v\|_{s}^{2}=\sum_{i=0}^{s} \int_{0}^{R}\left|v^{(i)}(x)\right|^{2} d x
$$

According to the boundary condition, we work on the solution space

$$
H_{0}^{1}(\Omega)=\left\{v \in H^{1}(\Omega) \mid v(0)=v(R)=0\right\},
$$

equipped with the inner product

$$
[u, v]=\int_{0}^{R} u^{\prime}(x) v^{\prime}(x) d x, \quad \text { for } u, v \in H_{0}^{1}(\Omega)
$$


and its associated norm $|\cdot|$. It is well known that the norm $|\cdot|$ is equivalent to the standard norm $\|\cdot\|_{1}$ in this space.

Let $p \in \mathbb{N}$. We will apply the multilevel augmentation method and anti-derivatives of wavelets of order $p$ to find numerical solutions of two-point boundary value problems with Dirichlet boundary conditions.

Assume that there exists a unique weak solution $u \in H_{0}^{1}(\Omega)$. To find numerical solutions, we propose the following steps:

1. The solution space $H_{0}^{1}(\Omega)$ is decomposed into orthogonal direct sum of subspaces. The anti-derivatives of the Daubechies wavelets are used to construct finite-dimensional subspaces.

2. For $n \in \mathbb{N}$, the multilevel method is applied to obtain the $n$th level solution by solving a linear system with matrix coefficients related to the anti-derivatives of the Daubechies wavelets.

3. To obtain a solution at a higher level, namely $(n+i)$ th level, the multilevel augmentation method is applied. By the algorithm to be presented, the computational time for solving the linear system is reduced since the dimension of the matrix coefficient is smaller.

Finally, this work is organized as follows. Section 2 gives an introduction to the antiderivatives of the Daubechies wavelets and the finite-dimensional subspaces of the solution space $H_{0}^{1}(\Omega)$. In Section 3, we describe the algorithm to find approximate solutions using the multilevel augmentation method. The optimal error estimates for the approximate solutions are proven in Section 4, while some numerical examples are demonstrated in Section 5. Conclusions and future work are discussed in Section 6.

\section{Bases for subspaces of $H_{0}^{1}(\Omega)$}

In this section, we will introduce the wavelets of order $p$ and their anti-derivatives. These functions form orthonormal bases for the finite-dimensional subspaces $S_{n}$ of the solution space $H_{0}^{1}(\Omega)$. More details can be found in [5] and [13].

To define the Daubechies wavelets, we consider two functions: the scaling function $\phi(x)$ and the wavelet function $\psi(x)$. The scaling function is obtained from the dilation equation. The wavelet function is defined from the scaling function. Details are described as follows.

Given a positive integer $p$, consider a sequence $\left\{c_{k}\right\}_{k \in \mathbb{Z}}$ satisfying

$$
\begin{aligned}
& c_{k}=0, \quad \text { for } k \notin\{0,1, \ldots, 2 p-1\}, \\
& \sum_{k} c_{k}=2, \\
& \sum_{k}(-1)^{k} k^{m} c_{k}=2, \quad \text { for } 0 \leq m \leq p-1, \\
& \sum_{k} c_{k} c_{k-2 m}=2 \delta_{0 m}, \quad \text { for } 1-p \leq m \leq p-1 .
\end{aligned}
$$

The scaling function $\phi(x)$ is the unique solution of the dilation equation

$$
\phi(x)=\sum_{k=0}^{2 p-1} c_{k} \phi(2 x-k),
$$

where $c_{k}$ satisfy the above four properties. 
Figure $1 \phi$ and $\psi$ for $p=2$.

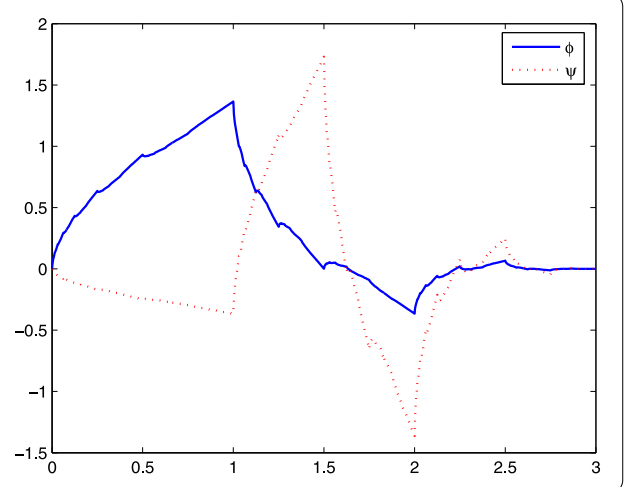

Let

$$
\widetilde{\psi}(x)=\sum_{k=0}^{2 p-1}(-1)^{k} c_{2 p-1-k} \phi(2 x-k) .
$$

The wavelet function $\psi(x)$ is defined by

$$
\psi(x)=\widetilde{\psi}(x-p+1)
$$

The graphs of $\phi$ and $\psi$ for $p=2$ are shown in Figure 1 .

Define

$$
\psi_{j k}(x)= \begin{cases}\phi(x-k), & j=-1 \\ \sqrt{2^{j}} \psi\left(2^{j} x-k\right), & j \geq 0\end{cases}
$$

The functions $\psi_{j k}$ with $j \geq-1$ and $(j, k) \in \mathbb{Z} \times \mathbb{Z}$ are called wavelets of order $p$. It is well known that the set of wavelets forms an orthonormal basis for $L^{2}(\mathbb{R})$.

For $j \geq-1$, define the index set $I_{j}$ such that

$$
k \in I_{j} \Longleftrightarrow \begin{cases}2-2 p \leq k \leq 2 p-2, & j=-1 \\ 2-2 p \leq k \leq 2^{j}(2 p-1)-1, & j \geq 0\end{cases}
$$

$\left\{\left.\psi_{j k}\right|_{\Omega} \mid j \geq-1, k \in I_{j}\right\}$ is a frame of $L^{2}(\Omega)$. That is, the $\operatorname{span}\left\{\left.\psi_{j k}\right|_{\Omega} \mid j \geq-1, k \in I_{j}\right\}$ consisting of all linear expansions is equal to $L^{2}(\Omega)$.

Next, we define the anti-derivatives of wavelets satisfying the Dirichlet boundary condition, namely,

$$
\Psi_{j k}(x)=\int_{0}^{x} \psi_{j k} d s-\frac{x}{R} \int_{0}^{R} \psi_{j k} d s, \quad \text { for } 0 \leq x \leq R .
$$

For $n \in \mathbb{N}_{0}$, we define a subspace $S_{n}$ by

$$
S_{n}=\operatorname{span}\left\{\Psi_{j k} \mid-1 \leq j<n, k \in I_{j}\right\}
$$

It is obvious that these subspaces are finite-dimensional subspaces of $H_{0}^{1}(\Omega)$ and they are nested, i.e., $S_{n} \subset S_{n+1}$. Since $\left\{\Psi_{j k} \mid-1 \leq j<n, k \in I_{j}\right\}$ is a frame for $S_{n}$, it spans $S_{n}$ but it 
Figure $2 \Psi_{00}, \Psi_{10}, \Psi_{11}, \Psi_{20}, \Psi_{21}, \Psi_{22}, \Psi_{23}$.

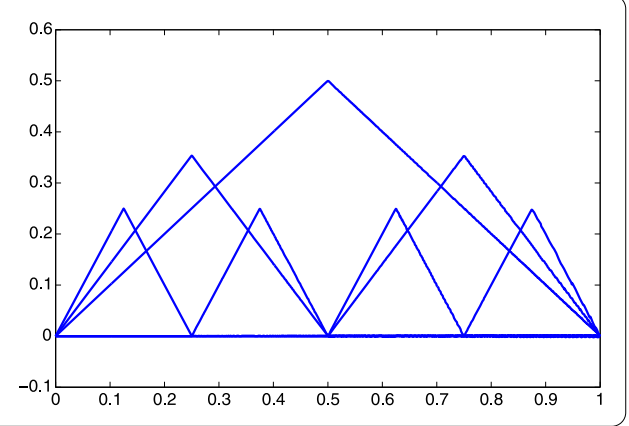

Figure $3 \Psi_{2,-1}, \ldots, \Psi_{2,10}$.

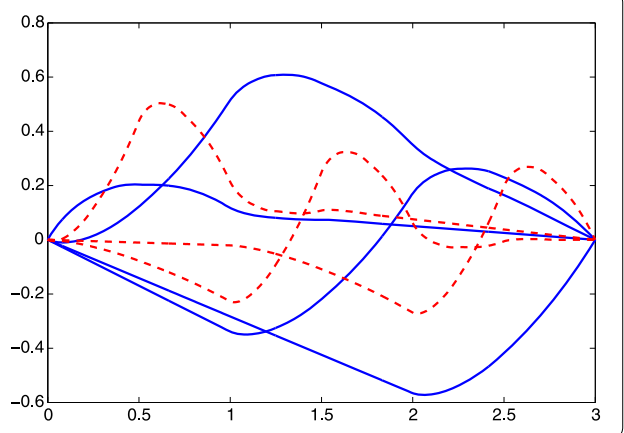

need not be linearly independent. Xu and Shann [13], introduced an index set $D_{j}$ such that $\left\{\Psi_{j k} \mid-1 \leq j<n, k \in D_{j}\right\}$ is a basis for $S_{n}$. The index set $D_{j}$ is defined by

$$
k \in D_{j} \Longleftrightarrow \begin{cases}2-2 p<k \leq 2 p-2, & j=-1, \\ 1-p \leq k \leq 2^{j}(2 p-1)-p, & j \geq 0 .\end{cases}
$$

For $p=1$, the basis functions $\left\{\Psi_{j k} \mid 0 \leq j<n, k \in D_{j}\right\}$ for $S_{n}$ are the piecewise linear hierarchical basis functions with uniform mesh size, $h=2^{-n}$. The graphs of $\Psi_{00}, \Psi_{10}, \ldots, \Psi_{22}$, and $\Psi_{23}$ are shown in Figure 2, while the graphs of $\Psi_{2,-1}, \ldots, \Psi_{2,10}$ are shown in Figure 3.

It should be noted that the set

$$
\left\{\Psi_{j k} \mid 0 \leq j<n, k \in D_{j}\right\}
$$

is an orthonormal basis for $S_{n}$ with the inner product $[\cdot, \cdot]$ of the Sobolev space $H_{0}^{1}(\Omega)$ but it is not orthonormal in $L^{2}(\Omega)$ when equipped with the standard inner product $(\cdot, \cdot)$. Since we require orthonormality in $L^{2}(\Omega)$ for our augmentation method, we apply the GramSchmidt process to obtain an orthonormal basis for $S_{n}$ with the standard inner product $(\cdot, \cdot)$ in $L^{2}(\Omega)$. Thus, the set

$$
\left\{\bar{\Psi}_{j k} \mid-1 \leq j<n, k \in D_{j}\right\}
$$

is defined as orthonormal basis for $S_{n}$ in the present method.

\section{Multilevel augmentation method algorithm}

In this section, we describe the multilevel augmentation method for solving boundary value problems. 
Let $u \in H_{0}^{1}(\Omega)$ be the weak solution of a given differential equation. Suppose that the variational form of the differential equation is

$$
a(u, v)=(f, v), \quad \text { for all } v \in H_{0}^{1}(\Omega) .
$$

Let the approximate solution $u_{n}$ of the given equation be

$$
u_{n}=\sum_{j=-1}^{n} \sum_{k \in D_{j}} \alpha_{j k} \bar{\Psi}_{j k} .
$$

The main idea is to determine the coefficients $\alpha_{j k}$ in such a way that $u_{n}$ behaves as if it were a weak solution on $S_{n}$, that is, $u_{n}$ satisfies the linear system of equations,

$$
\begin{aligned}
& a\left(u_{n}, \bar{\Psi}_{l m}\right)=\left(f, \bar{\Psi}_{l m}\right), \quad \text { for all }-1 \leq l<n, m \in D_{j}, \\
& \sum_{-1 \leq j<n} \sum_{k \in D_{j}} \alpha_{j k} a\left(\bar{\Psi}_{j k}, \bar{\Psi}_{l m}\right)=\left(f, \bar{\Psi}_{l m}\right), \quad \text { for all }-1 \leq l<n, m \in D_{j} .
\end{aligned}
$$

Let $\iota:(j, k) \mapsto i$ be the lexicographically enumerating function. That is,

$$
\iota(j, k) \leq \iota(l, m) \quad \text { if } j \leq l \text { or } j=l \text { and } k \leq m .
$$

We then obtain a linear system of the form $\mathbf{A}_{n} \mathbf{u}_{n}=\mathbf{f}_{n}$, where $\mathbf{A}_{n}$ is the coefficient matrix, $\mathbf{u}_{n}$ is the unknown column vector, and $\mathbf{f}_{n}$ is the column vector defined by

$$
\begin{aligned}
& \mathbf{A}_{n}=\left[a\left(\bar{\Psi}_{i}, \bar{\Psi}_{j}\right): i, j=1, \ldots, \operatorname{dim} S_{n}\right], \\
& \mathbf{u}_{n}=\left[\alpha_{j}: j=1, \ldots, \operatorname{dim} S_{n}\right], \\
& \mathbf{f}_{n}=\left[\left(f, \bar{\Psi}_{j}\right): j=1, \ldots, \operatorname{dim} S_{n}\right] .
\end{aligned}
$$

The approximate solution $u_{n}$ obtained in this way is called the $n$th multilevel solution of (1).

Next, we apply the augmentation method to approximate the next level solution, $u_{n+1}$. Suppose that $u_{n}$ is already solved. That is, the matrix representation $\mathbf{u}_{n}$ of $u_{n}$ satisfies the equation $\mathbf{A}_{n} \mathbf{u}_{n}=\mathbf{f}_{n}$. We augment the matrix $\mathbf{A}_{n}$ with submatrices $\mathbf{B}_{n}, \mathbf{C}_{n}$, and $\mathbf{D}_{n}$ where

$$
\begin{aligned}
& \mathbf{B}_{n}=\left[a\left(\bar{\Psi}_{i}, \bar{\Psi}_{j}\right): i=1, \ldots, \operatorname{dim} S_{n}, j=\operatorname{dim} S_{n}+1, \ldots, \operatorname{dim} S_{n+1}\right], \\
& \mathbf{C}_{n}=\left[a\left(\bar{\Psi}_{i}, \bar{\Psi}_{j}\right): i=\operatorname{dim} S_{n}+1, \ldots, \operatorname{dim} S_{n+1}, j=1, \ldots, \operatorname{dim} S_{n}\right], \\
& \mathbf{D}_{n}=\left[a\left(\bar{\Psi}_{i}, \bar{\Psi}_{j}\right): i, j=\operatorname{dim} S_{n}+1, \ldots, \operatorname{dim} S_{n+1}\right] .
\end{aligned}
$$

The coefficient matrix $\mathbf{A}_{n+1}$ corresponding to the $(n+1)$ st level is identified as

$$
\mathbf{A}_{n+1}=\left[\begin{array}{cc}
\mathbf{A}_{n} & \mathbf{B}_{n} \\
\mathbf{C}_{n} & \mathbf{D}_{n}
\end{array}\right] .
$$

Instead of finding the full ( $n+1)$ th level solution, $u_{n+1}$, from $\mathbf{A}_{n+1} \mathbf{u}_{n+1}=\mathbf{f}_{n+1}$ of size $\operatorname{dim} S_{n+1}$, we will approximate $u_{n+1}$ by decomposition the coefficient matrix $\mathbf{A}_{n+1}$ into the sum of an 
upper triangular matrix and a lower triangular matrix:

$$
\mathbf{A}_{n+1}=\left[\begin{array}{ll}
\mathbf{A}_{n} & \mathbf{B}_{n} \\
\mathbf{C}_{n} & \mathbf{D}_{n}
\end{array}\right]=\left[\begin{array}{cc}
\mathbf{A}_{n} & \mathbf{B}_{n} \\
0 & \mathbf{D}_{n}
\end{array}\right]+\left[\begin{array}{cc}
0 & 0 \\
\mathbf{C}_{n} & 0
\end{array}\right] .
$$

If the matrices $\mathbf{A}_{n}$ and $\mathbf{D}_{n}$ are nonsingular, there exists a (unique) vector $\mathbf{u}_{n, 1}$ satisfying

$$
\left[\begin{array}{cc}
\mathbf{A}_{n} & \mathbf{B}_{n} \\
0 & \mathbf{D}_{n}
\end{array}\right] \mathbf{u}_{n, 1}+\left[\begin{array}{cc}
0 & 0 \\
\mathbf{C}_{n} & 0
\end{array}\right]\left[\begin{array}{c}
\mathbf{u}_{n} \\
0
\end{array}\right]=\mathbf{f}_{n+1} .
$$

Let $u_{n, 1} \in S_{n+1}$ be the corresponding element of $\mathbf{u}_{n, 1}$. We call $u_{n, 1}$ the $(n+1)$ st multilevel augmentation solution of (1). The index $n$ refers to the initial level $n$, and 1 refer to a one step method to compute $u_{n+1}$ approximately. The linear system of $\operatorname{dim} S_{n+1}$ can be solved by considering two linear systems. One is the size of $\operatorname{dim} S_{n}$, and another is the size of $\operatorname{dim} S_{n+1}-\operatorname{dim} S_{n}=2^{n}(2 p-1)$.

In general, an approximation $u_{n, i+1}$ for $(n+i+1)$ st multilevel solution is defined by setting

$$
u_{n, 0}=u_{n} .
$$

Suppose that $u_{n, i}$ is already solved. We augment the matrix $\mathbf{A}_{n+i}$ with submatrices $\mathbf{B}_{n+i}$, $\mathbf{C}_{n+i}$, and $\mathbf{D}_{n+i}$ where

$$
\begin{aligned}
& \mathbf{B}_{n+i}=\left[a\left(\bar{\Psi}_{i}, \bar{\Psi}_{j}\right): i=1, \ldots, \operatorname{dim} S_{n+i}, j=\left(\operatorname{dim} S_{n+i}\right)+1, \ldots, \operatorname{dim} S_{n+i+1}\right], \\
& \mathbf{C}_{n+i}=\left[a\left(\bar{\Psi}_{i}, \bar{\Psi}_{j}\right): i=\left(\operatorname{dim} S_{n+i}\right)+1, \ldots, \operatorname{dim} S_{n+i+1}, j=1, \ldots, \operatorname{dim} S_{n+i}\right], \\
& \mathbf{D}_{n+i}=\left[a\left(\bar{\Psi}_{i}, \bar{\Psi}_{j}\right): i, j=\left(\operatorname{dim} S_{n+i}\right)+1, \ldots, \operatorname{dim} S_{n+i+1}\right] .
\end{aligned}
$$

The coefficient matrix $\mathbf{A}_{n+i+1}$ corresponding to the $(n+i+1)$ st level is identified as

$$
\mathbf{A}_{n+i+1}=\left[\begin{array}{ll}
\mathbf{A}_{n+i} & \mathbf{B}_{n+i} \\
\mathbf{C}_{n+i} & \mathbf{D}_{n+i}
\end{array}\right] .
$$

We split the coefficient matrix $\mathbf{A}_{n+i+1}$ and solve for $u_{n, i+1} \in S_{n+i+1}$ from the equation

$$
\left[\begin{array}{cc}
\mathbf{A}_{n+i} & \mathbf{B}_{n+i} \\
0 & \mathbf{D}_{n+i}
\end{array}\right] \mathbf{u}_{n, i+1}+\left[\begin{array}{cc}
0 & 0 \\
\mathbf{C}_{n+i} & 0
\end{array}\right]\left[\begin{array}{c}
\mathbf{u}_{n, i} \\
0
\end{array}\right]=\mathbf{f}_{n+i+1} .
$$

This completes the multilevel augmentation algorithm.

\section{Error analysis}

In this section, we analyze the convergence of the multilevel augmentation method conjunction with the anti-derivatives of the Daubechies wavelets. First, we consider the distance between the weak solution $u$ of (1) and the $n$th multilevel solution $u_{n} \in S_{n}$ obtained by the wavelets of order $p$. Theorem 4.1 in [13] stated that, for $u \in H_{0}^{1}(\Omega) \cap H^{s}(\Omega)$, there 
exists a positive constant $C$ such that

$$
\left\|u-u_{n}\right\|+2^{-n}\left\|u-u_{n}\right\|_{1} \leq C\left(2^{-n}\right)^{s}\|u\|_{s}, \quad 1 \leq s \leq p+1 .
$$

In particular, if we consider separately the distance between $u$ and $u_{n}$ with the standard $L^{2}$ norm $\|\cdot\|$, and the Sobolev norm $\|\cdot\|_{1}$, we obtain

$$
\begin{aligned}
& \left\|u-u_{n}\right\| \leq C\left(2^{-n}\right)^{s}\|u\|_{s}, \quad 1 \leq s \leq p+1, \\
& \left\|u-u_{n}\right\|_{1} \leq C\left(2^{-n}\right)^{s-1}\|u\|_{s}, \quad 1 \leq s \leq p+1 .
\end{aligned}
$$

The above estimations suggest that if we apply the wavelet of order $p$ and $u \in H_{0}^{1}(\Omega) \cap$ $H^{s}(\Omega)$, then the errors measured by the standard norm, and the Sobolev norm in $L^{2}$, decrease by the factors of $2^{p+1}$, and $2^{p}$, respectively, from $n$ to $n+1$ level.

Next, we consider the distance between the solution $u$ and the $(n+i)$ th multilevel augmentation solution, $u_{n, i}$, of (1). In the remaining section, we denote by $\mathcal{A}$ the operator corresponding to the matrix $\mathbf{A}$, and we denote by $\mathbf{u}$ the column matrix representing element $u$.

For $n \in \mathbb{N}$, if the inverse operators $\mathcal{A}_{n}^{-1}$ and $\mathcal{D}_{n}^{-1}$ exist, then the $(n+1)$ th multilevel augmentation solution $u_{n, i}$ exists. If there also exist an $N \in \mathbb{N}, \alpha>0$, and $\delta>0$ such that

$$
\left\|\mathcal{A}_{n}^{-1}\right\| \leq \alpha, \quad\left\|\mathcal{D}_{n}^{-1}\right\| \leq \delta, \quad \text { for } n \geq N
$$

and

$$
\lim _{n \rightarrow \infty}\left\|\mathcal{B}_{n}\right\|=\lim _{n \rightarrow \infty}\left\|\mathcal{C}_{n}\right\|=0
$$

the error for our method can be estimated as in the following theorem.

Theorem 1 (Error for multilevel augmentation method) Let $u \in H_{0}^{1}(\Omega)$ be the solution of (1). Suppose that there exist an $N \in \mathbb{N}$, and positive constants $\alpha$ and $\delta$ such that for $n \geq N$ the inverse operators $\mathcal{A}_{n}^{-1}, \mathcal{D}_{n}^{-1}$ exist and

$$
\left\|\mathcal{A}_{n}^{-1}\right\| \leq \alpha, \quad\left\|\mathcal{D}_{n}^{-1}\right\| \leq \delta,
$$

and

$$
\lim _{n \rightarrow \infty}\left\|\mathcal{B}_{n}\right\|=\lim _{n \rightarrow \infty}\left\|\mathcal{C}_{n}\right\|=0
$$

If $u \in H_{0}^{1}(\Omega) \cap H^{s}(\Omega)$, then there exist an $M \in \mathbb{N}$ and positive constant $c_{0}$ such that, for $n \geq M$ and $i \in \mathbb{N} \cup\{0\}$, we have the estimate

$$
\left\|u-u_{n, i}\right\| \leq c_{0} 2^{-2(n+i) s}\|u\|_{s}, \quad 1 \leq s \leq p+1
$$

Proof By the hypotheses on $\mathcal{A}_{n}^{-1}$ and $\mathcal{D}_{n}^{-1}$, we see that the $(n+i)$ th multilevel augmentation solution $u_{n+i}$ and the $(n+i)$ th multilevel augmentation solution $u_{n, i}$ exist for all $n \geq N$ and 
$i \in \mathbb{N} \cup\{0\}$. By Theorem 4.1 in [13], there exists a positive constant $C$ such that

$$
\left\|u-u_{n+i}\right\| \leq C\left(2^{-(n+i) s}\right)\|u\|_{s}, \quad 1 \leq s \leq p+1
$$

For $n \geq N$ and $i \in \mathbb{N}$, let $W_{n+i-1}$ be the orthogonal complement of $S_{n+i-1}$ in $S_{n+i}$. The element $u_{n, i} \in S_{n+i}$ can be uniquely written in the form

$$
u_{n, i}=u_{n, i-1}^{1}+v_{n, i-1}^{1},
$$

where $u_{n, i-1}^{1} \in S_{n+i-1}$, and $v_{n, i-1}^{1} \in W_{n+i-1}$. Since the column matrix $\mathbf{u}_{n, i}$ of $u_{n, i}$ satisfies the equation

$$
\left[\begin{array}{cc}
\mathbf{A}_{n+i-1} & \mathbf{B}_{n+i-1} \\
0 & \mathbf{D}_{n+i-1}
\end{array}\right] \mathbf{u}_{n, i}+\left[\begin{array}{cc}
0 & 0 \\
\mathbf{C}_{n+i-1} & 0
\end{array}\right]\left[\begin{array}{c}
\mathbf{u}_{n, i-1} \\
0
\end{array}\right]=\mathbf{f}_{n+i}
$$

it also satisfies

$$
\mathbf{A}_{n+i} \mathbf{u}_{n, i}=\mathbf{f}_{n+i}+\left[\begin{array}{cc}
0 & 0 \\
\mathbf{C}_{n+i-1} & 0
\end{array}\right]\left[\begin{array}{c}
\mathbf{u}_{n, i-1}^{1}-\mathbf{u}_{n, i-1} \\
0
\end{array}\right] .
$$

The $(n+i)$ th multilevel solution $u_{n+i} \in S_{n+i}$ satisfies the equation

$$
\mathbf{A}_{n+i} \mathbf{u}_{n+i}=\mathbf{f}_{n+i} .
$$

Subtracting (6) from (5), we have

$$
\mathbf{A}_{n+i}\left(\mathbf{u}_{n, i}-\mathbf{u}_{n+i}\right)=\left[\begin{array}{cc}
0 & 0 \\
\mathbf{C}_{n+i-1} & 0
\end{array}\right]\left[\begin{array}{c}
\mathbf{u}_{n, i-1}^{1}-\mathbf{u}_{n, i-1} \\
0
\end{array}\right] .
$$

Since $\mathbf{A}_{n+i}$ is nonsingular, we have the equation

$$
\mathbf{u}_{n, i}-\mathbf{u}_{n+i}=\mathbf{A}_{n+i}^{-1}\left[\begin{array}{cc}
0 & 0 \\
\mathbf{C}_{n+i-1} & 0
\end{array}\right]\left[\begin{array}{c}
\mathbf{u}_{n, i-1}^{1}-\mathbf{u}_{n, i-1} \\
0
\end{array}\right]
$$

Since $\lim _{n \rightarrow \infty}\left\|\mathcal{B}_{n}\right\|=\lim _{n \rightarrow \infty}\left\|\mathcal{C}_{n}\right\|=0$, there exist an $M \in \mathbb{N}$ and $c>0$ such that for $n \geq M$ and $i \in \mathbb{N}$,

$$
\left\|\mathcal{C}_{n+i-1}\right\| \leq \frac{2^{-s}}{3 \alpha}
$$

and

$$
\left\|u_{n, i-1}^{1}-u_{n, i-1}\right\| \leq c\left(2^{-(n+i-1)}\right)^{s}\|u\|_{s}, \quad 1 \leq s \leq p+1 .
$$

We have, for $n \geq M, i \in \mathbb{N}$, and $1 \leq s \leq p+1$,

$$
\left\|u_{n, i}-u_{n+i}\right\| \leq\left\|\mathcal{A}_{n+i}^{-1}\right\|\left\|\mathcal{C}_{n+i-1}\right\|\left\|u_{n, i-1}^{1}-u_{n, i-1}\right\| \leq \frac{c}{3}\left(2^{-(n+i)}\right)^{s}\|u\|_{s} .
$$


From (4) and (7), we conclude that

$$
\begin{aligned}
\left\|u-u_{n, i}\right\| & \leq\left\|u-u_{n+i}\right\|+\left\|u_{n, i}-u_{n+i}\right\| \\
& \leq C\left(2^{-(n+i) s}\right)\|u\|_{s}+\frac{c}{3}\left(2^{-(n+i) s}\right)\|u\|_{s} \\
& \leq\left(C+\frac{c}{3}\right)\left(2^{-(n+i) s}\right)\|u\|_{s} .
\end{aligned}
$$

The above theorem suggests that, if the solution $u \in H_{0}^{1}(\Omega) \cap H^{s}(\Omega)$, and we apply the multilevel augmentation method from level $n+i-1$ to $n+i$ by using the anti-derivatives wavelets of order $p$, the errors measured in $\|\cdot\|$ decrease by a factor of $2^{p+1}$. Thus the behaviors of the decreasing error obtained by the multilevel, and the multilevel augmentation methods, are in the same order.

\section{Examples}

In this section, we illustrate the efficiency of the multilevel augmentation method in conjunction with the anti-derivatives of Daubechies wavelets of order $p$ to find the numerical solutions of the following boundary value problem:

$$
-\left(q(x) u^{\prime}\right)^{\prime}+r(x) u=f(x), \quad \text { for } x \in(0, R),
$$

with the Dirichlet conditions

$$
u(0)=u(R)=0,
$$

where $R=2 p-1$. It should be noted that the general interval $(\alpha, \beta)$ can be changed to $(0, R)$ by the method of changing variables.

We assume that $f \in L^{2}(\Omega)$, the coefficients $q$ and $r$ are smooth in the closed interval $[0, R]$ with $q>0$ and $r \geq 0$.

The variational form of $(8)$ is

$$
a(u, v)=(f, v), \quad \text { for all } v \in H_{0}^{1}(\Omega),
$$

where $a(\cdot, \cdot)$ is the bilinear form defined by

$$
a(u, v)=\int_{0}^{R} q u^{\prime} v^{\prime}+\operatorname{ruv} d x
$$

Since $A(\cdot, \cdot)$ is continuous and coercive on $H_{0}^{1}(\Omega)$, by the Lax-Milgram lemma, there exists a unique weak solution $u \in H_{0}^{1}(\Omega)$ for (9).

Note that we can also apply the multilevel augmentation method in conjunction with the anti-derivatives of the Daubechies wavelets order $p$ for the cases with nonzero boundary conditions. For example, we consider the boundary value problem

$$
-\left(q(x) u^{\prime}\right)^{\prime}+r(x) u=f(x), \quad \text { for } x \in(\alpha, \beta),
$$


with the boundary conditions

$$
u(\alpha)=c, \quad u(\beta)=d .
$$

We notice that the linear function

$$
y(x)=\frac{\beta c-\alpha d}{\beta-\alpha}+\frac{d-c}{\beta-\alpha} x
$$

satisfies the boundary conditions, that is, $y(\alpha)=c$ and $y(\beta)=d$. Let $u=y+w$ be the weak solution of (11). Since $u(\alpha)=c$ and $u(\beta)=d, w(\alpha)=w(\beta)=0$. The variational form of the boundary value problem in this case is

$$
a(w, v)=\int_{\alpha}^{\beta} f v d x-a(y, v), \quad \text { for all } v \in H_{0}^{1}(\alpha, \beta)
$$

where $a(\cdot, \cdot)$ is the bilinear form

$$
a(w, v)=\int_{\alpha}^{\beta} q w^{\prime} v^{\prime}+r w v d x .
$$

Since $a(\cdot, \cdot)$ is continuous and coercive on $H_{0}^{1}(\alpha, \beta)$, by the Lax-Milgram lemma, there exists a unique weak solution $w \in H_{0}^{1}(\alpha, \beta)$ for (12).

Example 1 For the first example, we consider the two-point boundary value problem

$$
-\left(x u^{\prime}\right)^{\prime}+x^{2} u=x^{4}-x^{3}-4 x+1, \quad \text { for } x \in(0,1),
$$

with boundary conditions $u(0)=u(1)=0$.

The exact solution is $u(x)=x^{2}-x$. Here, we apply the Daubechies wavelets of order $p=1$ to solve the problem. Numerical results for each level (n) are shown in Table 1 . The column of $\left\|u-u_{n}\right\|$ presents the numerical results obtained by the standard multilevel method. When increasing the level of approximations, the norm of the $L^{2}$ error decreases by the factor of $2^{s}$ where $1 \leq s \leq 2$. The numerical results by the multilevel augmentation method starting from the second and the third levels are shown in the $\left\|u-u_{1, n-1}\right\|$ and $\left\|u-u_{2, n-2}\right\|$ columns, respectively. At the same level $n$, the $L^{2}$ errors are in the same order as those of the standard multilevel method, except that its values are slightly greater. These additional errors come from the augmentation part which can be seen from the proof of Theorem 1. It can be seen further that the error from the augmentation method is getting closer to the error from the standard multilevel method as $n$ becomes large.

Example 2 Consider the boundary value problem

$$
-\left(e^{-x} u^{\prime}\right)^{\prime}=\pi e^{-x}(\cos (\pi x)+\pi \sin (\pi x)), \quad \text { for } x \in(0,1),
$$

with boundary conditions, $u(0)=u(1)=0$.

The exact solution is $u=\sin (\pi x)$. Numerical results for $p=1$ are shown in Table 2 . The column of $\left\|u-u_{n}\right\|$ presents the numerical results obtained by the multilevel method. 
Table 1 Example 1: Numerical results for $p=1$

\begin{tabular}{cclll}
\hline $\boldsymbol{n}$ & $\boldsymbol{d i m} \boldsymbol{S}_{\boldsymbol{n}}$ & $\left\|\boldsymbol{u}-\boldsymbol{u}_{\boldsymbol{n}}\right\|$ & $\left\|\boldsymbol{u}-\boldsymbol{u}_{\mathbf{1}, \boldsymbol{n}-\mathbf{1}}\right\|$ & $\left\|\boldsymbol{u}-\boldsymbol{u}_{\mathbf{2}, \boldsymbol{n}-\mathbf{2}}\right\|$ \\
\hline 2 & 3 & $8.0368 \mathrm{e}-001$ & & \\
3 & 7 & $5.6412 \mathrm{e}-001$ & $5.7409 \mathrm{e}-001$ & \\
4 & 15 & $3.7341 \mathrm{e}-001$ & $3.9796 \mathrm{e}-001$ & $4.0020 \mathrm{e}-001$ \\
5 & 31 & $2.2508 \mathrm{e}-001$ & $2.7097 \mathrm{e}-001$ & $2.6985 \mathrm{e}-001$ \\
6 & 63 & $1.4568 \mathrm{e}-001$ & $1.7275 \mathrm{e}-001$ & $1.7293 \mathrm{e}-001$ \\
7 & 127 & $8.8603 \mathrm{e}-002$ & $1.1148 \mathrm{e}-001$ & $1.1143 \mathrm{e}-001$ \\
\hline
\end{tabular}

Table 2 Example 2: Numerical results for $p=1$

\begin{tabular}{ccllll}
\hline $\boldsymbol{n}$ & $\boldsymbol{\operatorname { d i m }} \boldsymbol{S}_{\boldsymbol{n}}$ & $\boldsymbol{\| u - u _ { \boldsymbol { n } } \|}$ & $\boldsymbol{\| u - \boldsymbol { u } _ { \mathbf { 1 } , \boldsymbol { n } - \mathbf { 1 } } \|}$ & $\left\|u-\boldsymbol{u}_{\mathbf{2}, \boldsymbol{n}-\mathbf{2}}\right\|$ & $\left\|u-\boldsymbol{u}_{\mathbf{3}, \mathbf{n}-\mathbf{3}}\right\|$ \\
\hline 2 & 3 & 1.6427 & & & \\
3 & 7 & 0.6045 & 0.6458 & & \\
4 & 15 & 0.2424 & 0.2488 & 0.2494 & \\
5 & 31 & 0.1124 & 0.1162 & 0.1162 & 0.1170 \\
6 & 63 & 0.0589 & 0.0607 & 0.0607 & 0.0606 \\
7 & 127 & 0.0309 & 0.0325 & 0.0325 & 0.0325 \\
8 & 255 & 0.0154 & 0.0161 & 0.0161 & 0.0161 \\
9 & 511 & 0.0085 & 0.0087 & 0.0087 & 0.0087 \\
\hline
\end{tabular}

Table 3 Example 2: Numerical results for $p=2$

\begin{tabular}{|c|c|c|c|}
\hline$n$ & $\operatorname{dim} S_{n}$ & $\left\|u-u_{n}\right\|$ & $\left\|u-u_{1, n-1}\right\|$ \\
\hline 0 & 4 & 1.6268 & \\
\hline 1 & 7 & 0.2097 & \\
\hline 2 & 13 & 0.0250 & 0.0341 \\
\hline 3 & 25 & 0.0030 & 0.0041 \\
\hline 4 & 49 & 0.0004 & 0.0005 \\
\hline
\end{tabular}

The $L^{2}$ error decreases by the factor of $2^{s}$ where $1 \leq s \leq 2$ as expected, and this agrees well with the theoretical results. The $L^{2}$ errors obtained by the multilevel augmentation method starting from levels 2,3 , and 4 are shown in the $\left\|u-u_{1, n-1}\right\|,\left\|u-u_{2, n-2}\right\|$, and $\left\|u-u_{3, n-3}\right\|$ columns, respectively. Their values are in the same order as the multilevel method.

To apply the Daubechies wavelets of order $p=2$, we change the interval $[0,1]$ to $[0,3]$. So we obtain the following boundary value problem:

$$
-\left(e^{-x} u^{\prime}\right)^{\prime}=\frac{\pi}{3} e^{-x}\left(\cos \left(\frac{\pi}{3} x\right)+\frac{\pi}{3} \sin \left(\frac{\pi}{3} x\right)\right), \quad \text { for } x \in(0,3)
$$

with conditions $u(0)=u(3)=0$.

The exact solution of this problem is $u(x)=\sin \left(\frac{\pi}{3} x\right)$. Numerical results from the wavelet basis of order $p=2$ are shown in Table 3. The rate of $L^{2}$ error convergence is faster than that of the case $p=1$. Here, the $L^{2}$ error is four times smaller than that of the previous error level. This agrees with the theoretical result that the $L^{2}$ error should decrease by a factor of $2^{s}$, where $1 \leq s \leq 3$.

Example 3 Consider the boundary value problem with nonzero boundary conditions

$$
u^{\prime \prime}-u^{\prime}=x^{2}-4 x+1, \quad \text { for } x \in(0,1)
$$

with boundary conditions, $u(0)=-1, u(1)=1$. 
Table 4 Example 3: Numerical results for $p=1$

\begin{tabular}{|c|c|c|c|c|c|}
\hline$n$ & $\operatorname{dim} S_{n}$ & $\left\|u-u_{n}\right\|$ & $\left\|u-u_{1, n-1}\right\|$ & $\left\|u-u_{2, n-2}\right\|$ & $\left\|u-u_{3, n-3}\right\|$ \\
\hline 2 & 3 & 0.4221 & & & \\
\hline 3 & 7 & 0.1993 & 0.2123 & & \\
\hline 4 & 15 & 0.0994 & 0.1051 & 0.1049 & \\
\hline 5 & 31 & 0.0502 & 0.0561 & 0.0555 & 0.0555 \\
\hline 6 & 63 & 0.0278 & 0.0303 & 0.0303 & 0.0302 \\
\hline
\end{tabular}

Table 5 Example 3: Numerical results for $p=2$

\begin{tabular}{llll}
\hline $\boldsymbol{n}$ & $\boldsymbol{\operatorname { d i m }} \boldsymbol{S}_{\boldsymbol{n}}$ & $\boldsymbol{\| u - \boldsymbol { u } _ { \boldsymbol { n } } \boldsymbol { \| }}$ & $\boldsymbol{\| u - \boldsymbol { u } _ { \mathbf { 1 } , \boldsymbol { n } - \mathbf { 1 } \boldsymbol { \| } }}$ \\
\hline 0 & 4 & 3.3770 & \\
1 & 7 & 0.2888 & \\
2 & 13 & 0.0351 & 0.0378 \\
3 & 25 & 0.0044 & 0.0047 \\
4 & 49 & 0.0007 & 0.0008 \\
\hline
\end{tabular}

The exact solution is $u=-\frac{x^{3}}{3}+x^{2}+x+\frac{e^{x}-3 e+2}{3(e-1)}$. Numerical results for $p=1$ are shown in Table 4. Column of $\left\|u-u_{n}\right\|$ presents the numerical results obtained by the multilevel method. The $L^{2}$ error is twice smaller than that of the previous error level, and agrees well with the theoretical results. The $L^{2}$ errors obtained by the multilevel augmentation method starting from levels $3,4,5$, and 6 are shown in the $\left\|u-u_{1, n-1}\right\|,\left\|u-u_{2, n-2}\right\|$, and $\left\|u-u_{3, n-3}\right\|$ columns, respectively. Their values are in the same order as the multilevel method.

Numerical results from the wavelet basis of order $p=2$ are shown in Table 5 . The rate of $L^{2}$ error convergence is faster than that of the case $p=1$. Here, the $L^{2}$ errors is eight times smaller than that of the previous error level.

\section{Conclusions}

This present work is our attempt to apply the multilevel augmentation method using the anti-derivatives of Daubechies wavelets for approximating two-point boundary value problems with Dirichlet boundary conditions. This method is extended from the multilevel augmentation method that uses polynomial wavelet basis. An error analysis has also been presented. The rate of convergence is by a factor of $2^{s}, 1 \leq s \leq p+1$, where $p$ is the Daubechies wavelet order. At the same level, the $L^{2}$ error of the multilevel augmentation method is greater than that of the multilevel method, but they are in the same order.

The difficulty of this approach is that the anti-derivatives of Daubechies wavelet cannot be expressed in explicit form. One is required to solve the dilation equation to obtain a wavelet basis in an implicit formula. Here, we have done this using a numerical approximation to obtain the basis function point by point. Also, it is not easy to extend this approach to problems in higher dimension.

The advantage of this method is that we need not solve the full linear system. The unknown coefficients from the previous level can be used to approximate additional unknowns in the next level. Thus, this method can reduce computational time and memory for storing matrix coefficients. Furthermore, by applying the general anti-derivatives of Daubechies wavelets, this method can be used to solve the boundary value problems with Neumann and mixed boundary conditions. Numerical experiments on these problems are in progress and will be reported elsewhere further. 
Competing interests

The authors declare that they have no competing interests.

\section{Authors' contributions}

All authors contributed equally to the writing of this paper. All authors read and approved the final manuscript.

\section{Author details}

${ }^{1}$ Department of Mathematics, Faculty of Science, Chiang Mai University, Chiang Mai, 50200, Thailand. ${ }^{2}$ Department of Mathematics, Faculty of Science, Kasetsart University, Bangkok, 10900, Thailand.

\section{Acknowledgements}

This research was supported by Chiang Mai University.

Received: 25 September 2014 Accepted: 8 April 2015 Published online: 23 April 2015

\section{References}

1. Chen, Z, Micchelli, CA, Xu, Y: A multilevel method for solving operator equations. J. Math. Anal. Appl. 262, 688-699 (2001)

2. Chen, Z, Wu, B, Xu, Y: Multilevel augmentation methods for differential equations. Adv. Comput. Math. 24(1-4), 213-238 (2006)

3. Chen, Z, Wu, B, Xu, Y: Multilevel augmentation methods for solving operator equations. Numer. Math. J. Chin. Univ. $14,31-55(2006)$

4. Chen, Z, Xu, Y, Yang, H: Multilevel augmentation methods for solving ill-posed operator equations. Inverse Probl. 22 155-174 (2006)

5. Daubechies, I: Orthonormal bases of compactly supported wavelets. Commun. Pure Appl. Math. 41, 909-996 (1988)

6. Glowinski, R, Lawton, W, Ravachol, M, Tenenbaum, E: Wavelet Solutions of Linear and Nonlinear Elliptic, Parabolic and Hyperbolic Problems in One Space Dimension. Computing Methods in Applied Sciences and Engineering, pp. 55-120. SIAM, Philadelphia (1990)

7. Siraj-ul-Islam, Aziz, I, Al-Fahid, AS, Shah, A: A numerical assessment of parabolic partial differential equations using Haar and Legendre wavelets. Appl. Math. Model. 37, 9455-9481 (2013)

8. Siraj-ul-Islam, Aziz, I, Sarler, B: The numerical solution of second-order boundary value problems by collocation with Haar wavelets. Math. Comput. Model. 52, 1577-1590 (2010)

9. Amaratunga, K, Williams, JR, Qian, S, Weiss, J: Wavelet-Galerkin solutions for one dimensional partial differential equations, IESL Technical report, 9205, 2703-2716 (1994)

10. Jianhua, S, Xuming, Y, Biquan, Y, Yuantong, S: Wavelet-Galerkin solutions for differential equations. Wuhan Univ. J. Nat. Sci. 3(4), 403-406 (1998)

11. Mishra, V, Sabina: Wavelet Galerkin solutions of ordinary differential equations. Int. J. Math. Anal. 5(9), 407-424 (2011)

12. Qian, S, Weiss, J: Wavelets and the numerical solution of boundary value problems. Appl. Math. Lett. 6(1), 47-52 (1993)

13. Xu, JC, Shann, WC: Galerkin-wavelet methods for two point boundary value problems. Numer. Math. 63, 123-144 (1992)

\section{Submit your manuscript to a SpringerOpen ${ }^{\circ}$ journal and benefit from:}

- Convenient online submission

- Rigorous peer review

- Immediate publication on acceptance

- Open access: articles freely available online

- High visibility within the field

- Retaining the copyright to your article 